\title{
Measurement of the temperature-dependent speed of sound and change in Grüneisen parameter of tissue-mimicking materials
}

\author{
Marina Bakaric* ${ }^{*}$, Aoife Ivory ${ }^{\dagger}$, Bajram Zeqiri ${ }^{\dagger}$, Ben T. Cox*, Bradley E. Treeby* \\ ${ }^{*}$ Department of Medical Physics and Biomedical Engineering, University College London, London, UK \\ ${ }^{\dagger}$ Centre for Chemical, Environmental and Medical Science, National Physical Laboratory, Teddington, UK \\ †Email: marina.bakaric.16@ucl.ac.uk
}

\begin{abstract}
Photoacoustic thermometry is a rapidly emerging technique for non-invasive temperature monitoring. The temperature dependence of the Grüneisen parameter of tissues leads to changes in the recorded photoacoustic signal amplitude with temperature. In order to assess the material's suitability for a photoacoustic thermometry phantom, its temperature-dependent speed of sound and Grüneisen parameter must be known. Agarbased phantoms, copolymer-in-oil, gel wax, PVCP, silicone and water were thus characterised in a newly developed photoacoustic thermometry setup and the results presented for temperatures between 22 and $50{ }^{\circ} \mathrm{C}$. This information provides a valuable resource for the future development of tissue-mimicking materials with properties suitable for applications in photoacoustic thermometry.
\end{abstract}

Index Terms-metrology, phantoms, photoacoustics, thermometry, ultrasound

\section{INTRODUCTION}

Photoacoustic (PA) thermometry is a rapidly emerging technique for non-invasive temperature monitoring that exploits the temperature dependence of the Grüneisen parameter $\Gamma$ of tissues which leads to changes in the recorded photoacoustic signal amplitude with temperature and can thus be used to determine the temperature of that tissue [1]. In order to assess the accuracy, robustness and applicability of photoacoustic thermometry in different experimental scenarios, a stable phantom material with known properties is needed. This can also be used to tackle other challenges encountered in new imaging and therapeutic modalities, such as quality assurance [2].

Some of the requirements for real-time PA thermometry include correcting for temperature-induced changes in the speed of sound required for image reconstruction, as well as performing the measurements in the linear regime of the Grüneisen parameter dependency on temperature, ie. over a limited temperature range [3]. These thus determine the main properties of interest required to assess the suitability of phantoms for photoacoustic thermometry. However, current literature lacks information on the temperature dependency of the relevant phantom properties.

In this paper, we investigate the temperature-dependent speed of sound and Grüneisen parameter for some com-

This work was supported in part by the Engineering and Physical Sciences Research Council, grant numbers EP/L020262/1 and EP/P008860/1. monly used tissue-mimicking materials (TMMs) for photoacoustic and ultrasound imaging, namely agar-based phantoms, copolymer-in-oil, gel wax, PVCP, silicone, and water. For this purpose, a newly developed measurement setup is presented, capable of measuring the desired properties between 22 and $50^{\circ} \mathrm{C}$.

\section{MATERIALS AND METHODS}

\section{A. Tissue-mimicking materials}

The tissue-mimicking materials (TMMs) included in the study were agar-based materials [4], [5], copolymer-in-oil [6], gel wax [7], polyvinyl chloride plastisol (PVCP) [8] and silicone [9]. For each TMM, planar samples of $10 \pm 5$ $\mathrm{mm}$ thickness and $60 \mathrm{~mm}$ diameter were prepared with an embedded T-type thermocouple (5TC-TT-TI-36-1M-SMP-M IEC PFA-insulated, Omega Engineering Limited, Manchester, U.K.). The fabrication protocols were adopted from the literature, however, for the purpose of this study, acoustic scatterers were omitted and specialised black dyes were added to the base material in order to improve the signal-to-noise ratio. A summary of the base materials and dyes used in the fabrication of the phantoms, along with their relevant acoustic properties can be found in Table I.

\section{B. Experimental setup}

The experimental setup (Fig. 1) was comprised of a 1064 nm Q-Switched Nd:YAG laser (Ultra, Big Sky Laser Technologies, Bozeman, MT, USA) for illumination, a temperaturecontrolled water bath and a broadband PVDF receiver with a 5 $\mathrm{mm}$ active element and centre frequency of $14 \mathrm{MHz}$ (PA1075, Precision Acoustics Ltd., Dorchester, U.K.).

The laser light was delivered using an optical fibre with a numerical aperture of approximately $12^{\circ}(1 . a)$ and uniform illumination of the sample achieved using a lens (1.b), spreading the beam diameter to $30 \mathrm{~mm}$. The pulse duration, energy, and repetition frequency were $5.6 \mathrm{~ns}, 45 \mathrm{~mJ}$, and $20 \mathrm{~Hz}$, respectively. For safety purposes, the entire setup was placed in a black enclosure. The water bath consisted of a stainless steel tank (2.c) with inner dimensions of $150 \mathrm{~mm} \times 120 \mathrm{~mm}$ $\times 50 \mathrm{~mm}$ (height $\times$ width $\times$ length) and water heaters (2.d). The tank contained an optical window $(31 \mathrm{~mm} \times 31 \mathrm{~mm}$ BK7 window 1/4 wave $50 \mathrm{~mm}$ SQ NIR II, Edmund Optics 
TABLE I: Base materials and dyes used for the fabrication of the tissue-mimicking materials and their speed of sound $c$ and density $\rho$ as reported in the literature.

\begin{tabular}{|c|c|c|c|c|c|}
\hline TMM & Ref. & Base & Dye & c $\left(\mathrm{ms}^{-1}\right)$ & $\rho\left(\mathrm{kgm}^{-3}\right)$ \\
\hline Agar & [4], [5] & $\begin{array}{l}78.83 \% \text { water, } 11.21 \% \text { glycerol, } \\
3 \% \text { agar, } 0.95 \% 3-\mu \mathrm{m} \mathrm{Al}_{2} \mathrm{O}_{3} \text {, } \\
0.88 \% 0.3-\mu \mathrm{m}_{2} \mathrm{O}_{3}\end{array}$ & $\begin{array}{l}\text { India ink (Pelikan Vertriebsgesellschaft } \\
\text { mbH \& Co. KG, Hannover, Germany) }\end{array}$ & $1536.7 \pm 8.7$ & $1050 \pm 10.0$ \\
\hline Copolymer-in-oil & {$[6]$} & $12 \%$ copolymer oil $+3 \%$ LDPE & $\begin{array}{l}\text { Caligo safe wash relief ink, } \\
\text { Cranfield Colours, Cwmbran, UK }\end{array}$ & $1459 @ 3 \mathrm{MHz}$ & $900 \pm 5.0$ \\
\hline Gel wax & [7] & $\begin{array}{l}\text { FF1 003, Mindsets Online, } \\
\text { Waltham Cross, UK }\end{array}$ & $\begin{array}{l}\text { Caligo safe wash relief ink, } \\
\text { Cranfield Colours, Cwmbran, UK }\end{array}$ & $1445 \pm 2.7$ & $850 \pm 5.0$ \\
\hline PVCP & {$[8]$} & $\begin{array}{l}\text { Lure Flex Firm, } \\
\text { Lure Factors, Doncaster, UK }\end{array}$ & $\begin{array}{l}\text { Black Plastic Color } \\
\text { Lure Factors, Doncaster, UK }\end{array}$ & $1400 @ 3 \mathrm{MHz}$ & $1000 \pm 5.0$ \\
\hline Silicone & [9] & $\begin{array}{l}\text { Polytek PlatSil SiliGlass } \\
\text { MB Fibreglass, Newtownabbey, UK }\end{array}$ & $\begin{array}{l}\text { Polycraft Black Silicone Pigment } \\
\text { MB Fibreglass, Newtownabbey, UK }\end{array}$ & $1030 @ 7 \mathrm{MHz}$ & $1070 \pm 30$ \\
\hline
\end{tabular}

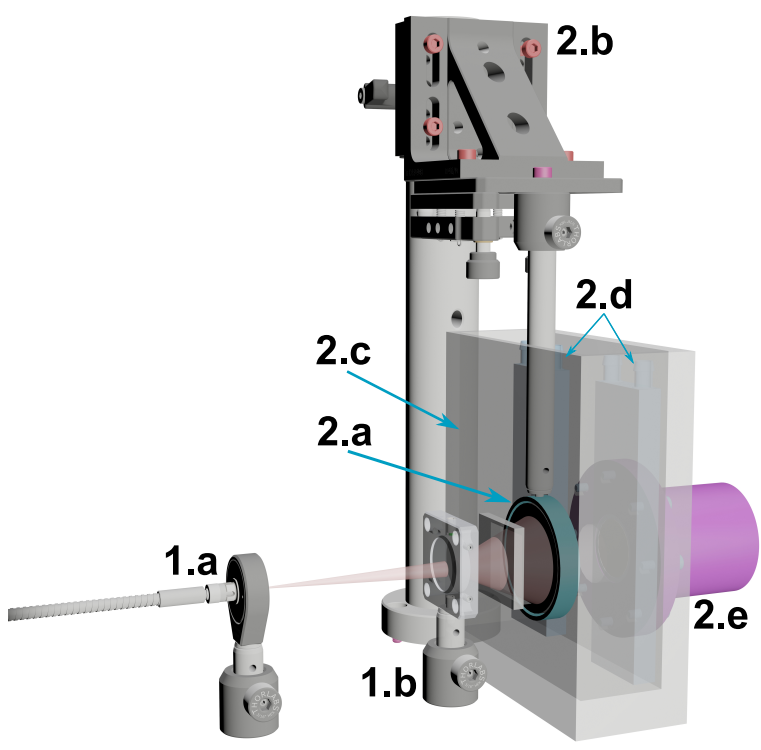

Fig. 1: Experimental setup comprising of a $1064 \mathrm{~nm}$ Ultra laser for illumination (1) and a temperature-controlled water bath (2). The light was delivered using an optical fibre (1.a) and uniform illumination of the sample achieved using a lens (1.b). The sample was held in a 3D printed holder (2.a), mounted to an optical post assembly with a 2-axis tilt (2.b) used for alignment and immersed in the water tank (2.c) with heaters (2.d). The generated photoacoustic signals were acquired using a broadband PVDF receiver with a $5 \mathrm{~mm}$ active element, which was held in a 3D printed transducer holder (2.e).

Ltd., York, U.K.) at the front and a circular opening $(\varnothing=23$ $\mathrm{mm}$ ) for the transducer at the back. For the measurements, the tank was filled with degassed deionised water. The sample was placed in a circular 3D printed sample holder (2.a) and mounted to an optical post assembly with a 2-axis tilt (2.b) used for alignment. The sample was immersed in the water and aligned for maximum signal.

The generated photoacoustic plane waves were recorded using the PVDF receiver which was held in an SLS nylon
3D printed holder (2.e). The holder attached to the tank from the rear side using eight hex socket cap screws and a silicone gasket providing a leak-proof attachment. The PVDF receiver was connected to an oscilloscope (DSO-X 3024A, Agilent Technologies, PaloAlto, CA, USA) using a submersible hydrophone preamplifier that buffers the signal and provides a $50 \mathrm{Ohm}$ source, which is powered by a DC coupler (Precision Acoustics Ltd., Dorchester, U.K.). Signals were digitised using the oscilloscope with a sampling frequency of $200 \mathrm{MHz}$ and 128 averages, and acquired every 1 degree between 22 and 50 degrees Celsius during both heating and cooling. The experiments were repeated 5 times in order to characterise the repeatability of the measurements.

The water (and thus sample) heating was achieved using a thermostat with an external circulation (ECO RE415S Silver thermostat, Lauda Dr. R. Wobser GmbH, Lauda-Königshofen, Germany) connected to two aluminium heat sinks immersed in the tank (2.d). An application for control of the various parts of the measurement process was built using LabVIEW (National Instruments, Austin, TX, USA). The temperature control was implemented using LabVIEW's in-built PID controller virtual instrument. The communication between the application and the external instruments was made via a USB interface.

In order to verify the acquired data for the TMMs and characterise the newly developed experimental setup, measurements were repeated using degassed deionised water as a reference material. For this purpose, the sample holder was removed from the bath and an OPO system (SpitLight 600, Innolas, Krailling, Germany) providing $6 \mathrm{~ns}$ pulses at $30 \mathrm{~Hz}$ tuned to the water absorption peak at $1470 \mathrm{~nm}$ was used for the generation of photoacoustic signals.

\section{Data analysis}

The data acquired using the setup described above was analysed offline using a Matlab script (R2018a, MathWorks, Massachusetts, USA). The recorded signals for each sample and temperature were first averaged and their DC offset removed. The peak values of the averaged signals were then extracted, and a time-of-flight method used to calculate the speed of sound in the samples.

a) Bulk speed of sound: The temperature-dependent bulk speed of sound (group velocity) of the samples was determined 
using the time of flight method [10]. The time between the laser trigger and the maximum peak in the photoacoustic signal was used in conjunction with the known temperaturedependent speed of sound in distilled water [11]. The speed of sound of the sample $\left(c_{\text {sample }}\right)$ was then calculated using the relation:

$$
c_{\text {sample }}=d_{\text {sample }}\left(t-\frac{d_{\text {water }}}{c_{\text {water }}}\right)^{-1}
$$

where $d_{\text {sample }}$ is the sample thickness, $t$ is the time of arrival of the photoacoustic signal, $c_{\text {water }}$ is the propagation speed in water and $d_{\text {water }}$ the water path length, ie. the distance between the sample and the receiver. The thickness of the samples was measured using a digital caliper with a resolution of $\pm 0.01 \mathrm{~mm}$ (Mitutoyo, Tokyo, Japan). The measurements were repeated five times per sample with a maximum standard deviation of $0.1 \mathrm{~mm}$. For water measurements, Eq. (1) reduces to $c_{\text {water }}=\frac{d_{\text {water }}}{t}$ where $d_{\text {water }}$ was taken to be equal to the length of the water tank.

b) Photoacoustic conversion efficiency: The photoacoustic conversion efficiency is the product of $\mu_{a}$ and $\Gamma$ [12]. This efficiency is reported instead of the Grüneisen parameter as for all solid samples the optical absorption coefficient did not show a strong temperature dependency. For water, a 5\% increase in the optical absorption coefficient was calculated over the measurement range. The optical absorption coefficient was calculated by performing curve-fitting to the exponential decay in the photoacoustic signal, for which only the knowledge of $c_{\text {sample }}$ and the temporal resolution is needed. Thus the recorded photoacoustic signals were used to retrieve the temperature-dependent change in $\mu_{a} \Gamma$ by extracting the peak values of the averaged signals and plotting against the thermocouple temperature data.

\section{RESUlTS AND DISCUSSION}

The temperature-dependent speed of sound of water, agarbased phantoms, copolymer-in-oil, gel wax, PVCP and silicone phantoms are presented in Fig. 2.

It can be seen that the measured temperature-dependent speed of sound of water shows a consistent offset of approximately $5 \mathrm{~ms}^{-1}$, while for the solid samples the values measured at the baseline temperature of the measurement are similar to the literature data for the used TMMs (Table I). These discrepancies can be contributed mostly to the uncertainty in sample thickness and water path length measured using a caliper. In order to reduce these errors, future studies should employ a broadband through-transmission method [13] to evaluate sample thickness by using the reflected signals from the front and rear surface of the samples.

The changes in the PA conversion efficiency of the tissuemimicking samples and water over the temperature range from $22{ }^{\circ} \mathrm{C}$ to $50^{\circ} \mathrm{C}$ are presented in Fig. 3. The exception are gel wax phantoms, for which the maximum measurement temperature was $40^{\circ} \mathrm{C}$ due to their low melting point compared to the other materials. The results are an average of five measurement repeats and are normalised to the amplitude at $22{ }^{\circ} \mathrm{C}$. The error bars are omitted for clarity, with coefficients (a) Water

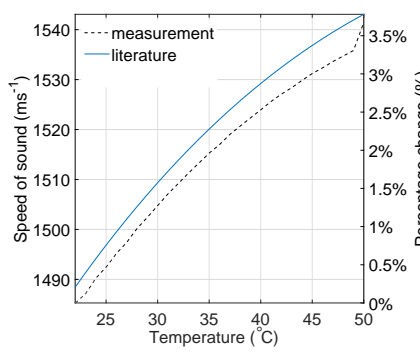

(c) Copolymer-in-oil

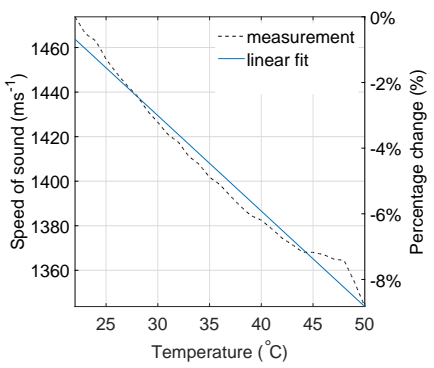

(e) PVCP
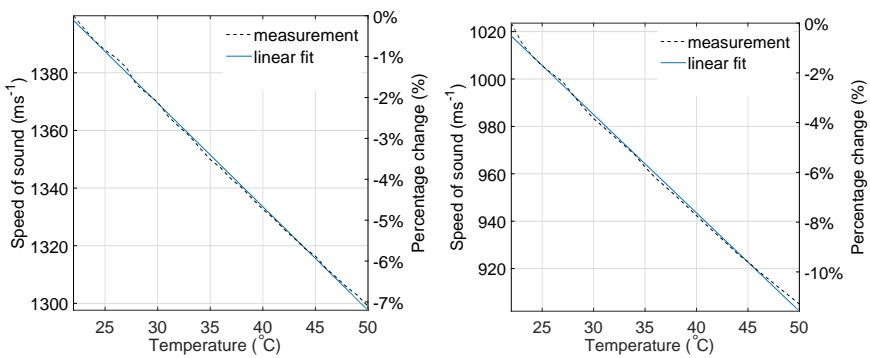

Fig. 2: Temperature-dependent speed of sound of (a) water, (b) agar-based phantoms, (c) copolymer-in-oil, (d) gel wax, (e) PVCP and (f) silicone phantoms.

of variation $<1.5 \%$ for all tested materials. The literature data for the temperature-dependent Grüneisen parameter of water is presented for reference, and was calculated using the approximation $\Gamma(T)=0.0053 T+0.0043$ derived from the definition of the Grüneisen parameter:

$$
\Gamma=\frac{\beta c^{2}}{C_{p}}
$$

and knowledge of the temperature dependence of the speed of sound, volume expansion thermal coefficient and specific heat under constant volume for water and aqueous solutions [14].

It can be seen that the measured temperature-dependent change in the photoacoustic conversion efficiency of water corresponds closely to the literature data for the Grüneisen parameter of water within the measurement temperature range, which increases by approximately $4 \%$ per degree Celsius.

The results for the TMMs can be sorted into three distinct categories. The first one are the agar-based phantoms. As their content is mostly water, the photoacoustic signal amplitudes generated in these samples increase with temperature accordingly. Copolymer-in-oil phantoms and PVCP, on the other 


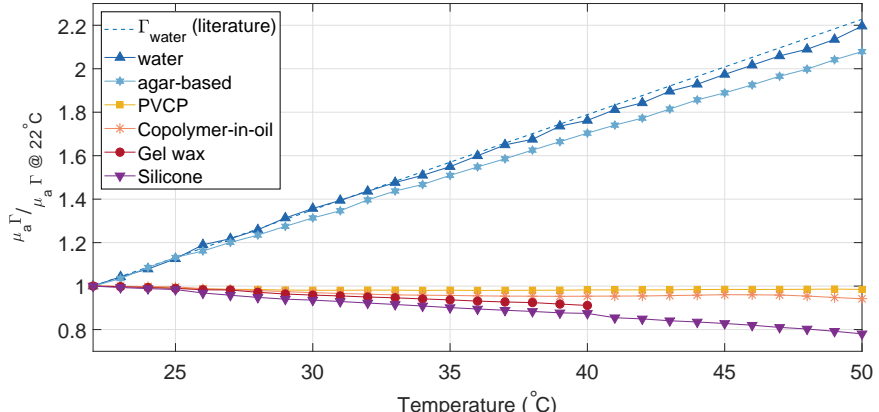

Fig. 3: The normalised temperature-dependent change in photoacoustic conversion efficiency $\mu_{a} \Gamma$ generated in phantoms over the temperature range from 22 to $50^{\circ} \mathrm{C}$. The literature data for the temperature-dependent Grüneisen parameter of water is presented for reference.

hand, do not seem to exhibit any significant change in the PA conversion efficiency. Although their speed of sound was measured to decrease by $7-8 \%$ within the measurement range, the remaining material properties contributing to the Grüneisen parameter (Eq. (2)) and their dependency on temperature are unknown. The change in the PA conversion efficiency in gel wax and silicone phantoms exhibits a decreasing trend with temperature. This is expected due to their oil content arising from the base material and dye used, for which it is known the speed of sound to decrease with temperature [15].

\section{CONCLUSION}

The temperature-dependent speed of sound and change in photoacoustic conversion efficiency for selected tissuemimicking materials is presented. This information forms a valuable resource for the future development of TMMs with properties suitable for applications in photoacoustic thermometry.

\section{REFERENCES}

[1] P. Beard, "Biomedical photoacoustic imaging," Interface focus, vol 1(4), pp. 602-631, 2011.

[2] J. R. Cook, R. R. Bouchard, and S. Y. Emelianov, "Tissue-mimicking phantoms for photoacoustic and ultrasonic imaging," Biomed. Opt. Express, vol. 2(11), pp. 3193--3206, 2011.

[3] E. Petrova, S. Ermilov, R. Su, V. Nadvoretskiy, A. Conjusteau, and A. Oraevsky, "Using optoacoustic imaging for measuring the temperature dependence of Grüneisen parameter in optically absorbing solutions," Opt. Express, vol. 21(21), pp. 25077--25090, 2013.

[4] International Electrotechnical Commission [IEC], "Medical electrical equipment: Part 2--37. Particular requirements for the basic safety and essential performance of ultrasonic medical diagnostic and monitoring equipment," IEC Publication 60601, Edition 2, Geneva: 2007a.

[5] A. Rabell-Montiel, T. Anderson, S. D. Pye, and C. M. Moran,"Attenuation coefficients of the individual components of the International Electrotechnical Commission agar tissue-mimicking material," Ultrasound Med. Biol., vol. 44(11), pp. 2371-2378, 2018.

[6] A. M. Ivory, A. Shah, S. Rajagopal, and B. Zeqiri, "Development and investigation of the acoustic properties of tissue-mimicking materials for photoacoustic imaging techniques," Proc. IEEE, 2019, in press.

[7] E. Maneas, W. Xia, O. Ogunlade, M. Fonseca, D. I. Nikitichev, A. L. David, et al., "Gel wax-based tissue-mimicking phantoms for multispectral photoacoustic imaging," Biomed. Opt. Express, vol. 9(3), pp. 1151-1163, 2018.
[8] M. Bakaric, P. Miloro, B. Zeqiri, B. T. Cox and B. E. Treeby, "The effect of curing temperature and time on the acoustic and optical properties of PVCP," Trans. Ultrason., Ferroelectr., Freq. Control, in press.

[9] K. Zell, J. I. Sperl, M. W. Vogel, R. Niessner, and C. Haisch, "Acoustical properties of selected tissue phantom materials for ultrasound imaging," Phys. Med. Biol., vol. 52(20), pp. N475, 2007.

[10] D. K. Hsu, and M. S. Hughes, "Simultaneous ultrasonic velocity and sample thickness measurement and application in composites," $\mathbf{J}$. Acoust. Soc. Am., vol. 92(2), pp. 669-675, 1992.

[11] W. Marczak, "Water as a standard in the measurements of speed of sound in liquids," J. Acoust. Soc. Am., vol. 102(5), pp. 2776-2779, 1997.

[12] S. M. Nikitin, T. D. Khokhlova, I. M. and Pelivanov, "Temperature dependence of the optoacoustic transformation efficiency in ex vivo tissues for application in monitoring thermal therapies," J. Biomed. Opt., vol. 17(6), pp. 061214, 2012.

[13] B. Zeqiri, W. Scholl, and S. P. Robinson, "Measurement and testing of the acoustic properties of materials: a review," Metrologia, vol. 47(2), pp. S156, 2010.

[14] L. V. Wang, and H. Wu, "Biomedical optics: principles and imaging," John Wiley \& Sons, 2012.

[15] C. A. Miles, G. A. J. Fursey, and R. C. D. Jones, "Ultrasonic estimation of solid/liquid ratios in fats, oils and adipose tissue," J. Sci. Food Agric., vol. 36(3), pp. 215-228, 1985. 